\title{
Diffuse correlation tomography in the transport regime: A theoretical study of the sensitivity to Brownian motion
}

\author{
Ugo Tricoli, ${ }^{1,{ }^{*}}$ Callum M. Macdonald, ${ }^{1}$ Turgut Durduran, ${ }^{2,3}$ Anabela Da Silva, ${ }^{1}$ and Vadim A. Markel ${ }^{1, \dagger}$ \\ ${ }^{1}$ Aix Marseille Univ, CNRS, Centrale Marseille, Institut Fresnel, Marseille 13013, France \\ ${ }^{2}$ ICFO-Institut de Ciències Fotòniques, The Barcelona Institute of Science and Technology, 08860 Castelldefels (Barcelona), Spain \\ ${ }^{3}$ Institució Catalana de Recerca i Estudis Avançats (ICREA), 08015 Barcelona, Spain
}

(Received 23 October 2017; published 20 February 2018)

\begin{abstract}
Diffuse correlation tomography (DCT) uses the electric-field temporal autocorrelation function to measure the mean-square displacement of light-scattering particles in a turbid medium over a given exposure time. The movement of blood particles is here estimated through a Brownian-motion-like model in contrast to ordered motion as in blood flow. The sensitivity kernel relating the measurable field correlation function to the mean-square displacement of the particles can be derived by applying a perturbative analysis to the correlation transport equation (CTE). We derive an analytical expression for the CTE sensitivity kernel in terms of the Green's function of the radiative transport equation, which describes the propagation of the intensity. We then evaluate the kernel numerically. The simulations demonstrate that, in the transport regime, the sensitivity kernel provides sharper spatial information about the medium as compared with the correlation diffusion approximation. Also, the use of the CTE allows one to explore some additional degrees of freedom in the data such as the collimation direction of sources and detectors. Our results can be used to improve the spatial resolution of DCT, in particular, with applications to blood flow imaging in regions where the Brownian motion is dominant.
\end{abstract}

DOI: 10.1103/PhysRevE.97.022408

\section{INTRODUCTION}

Over the last two decades, noninvasive tomographic optical imaging of multiply scattering biological tissues has attracted significant attention [1-3]. A relatively novel development in this field of research is diffuse correlation tomography (DCT). This family of methods utilizes the electric-field autocorrelation of the diffuse laser speckles to estimate the mean-square displacement of the scatterers inside the medium over a given period of time. This, in turn, can be related to the microvascular blood flow index [4-6] and to other types of internal motion of particles.

The vast majority of the proposed DCT implementations rely on the use of the diffusion equation as the mathematical model for propagation of the field correlations. Correspondingly, the technique has been applied under the conditions when the diffusion approximation to the more fundamental correlation transport equation (CTE) is valid. In this case, some information about the medium is lost due to the near-isotropy of radiation in the diffuse propagation regime [6]. In the mesoscopic scattering regime, it is possible to increase the information content of the data by exploring some additional degrees of freedom such as polarization or the source and detector collimation directions. However, this requires the use of the CTE as the mathematical model to capture correctly the strong anisotropy of the light scattering. Utilization of the

\footnotetext{
*ugo.tricoli@fresnel.fr

${ }^{\dagger}$ vmarkel@pennmedicine.upenn.edu; Currently with the Department of Radiology, University of Pennsylvania, Philadelphia, Pennsylvania 19104, USA.
}

CTE in the context of DCT is the main subject of this paper. Correspondingly, we consider samples of relatively smaller size than in the traditional implementations. However, multiple scattering is still important for us.

A technique related to DCT that relies on single scattering only is known as dynamic light scattering. This approach is based on measuring temporal fluctuations of the scattered light that are effected by the motion of particles within the medium. Dynamic light scattering has been successfully applied to characterize dilute media [7]. However, in more dense and turbid media, multiple scattering sets in. The theory of correlation propagation in the multiple-scattering regime was developed in Refs. [8-10] and is known as diffusing-wave spectroscopy (DWS). It was shown that, within the diffusion approximation and considering only uncorrelated single-scattering events, the detected correlation function is equal to the angle-averaged signal for single scattering elevated to the power equal to the number of random-walk steps along the photon path [4-6]. DWS was highly successful in modeling uniform, concentrated systems. Still, the biggest challenge in applying this theory to practical biomedical imaging has been the lack of a quantitative mathematical model for propagation of correlations carrying the spatial information inside the medium, especially when it is nonuniform. This problem was solved in DCT, wherein the correlation diffusion equation was introduced as an approximation to the CTE, enabling the development of practical imaging modalities [4-6]. Thus, the CTE can be seen as an integro-differential equation formulation of DWS which remains valid in the transport regime [6]. To increase the depth penetration and resolution, DCT employs point sources rather than wide-field illumination [4-6,11] and can be operated as a three-dimensional fully tomographic modality [12] by 
using many source-detector pairs, similar to the diffuse optical tomography.

Another related technique is laser speckle contrast imaging, which can produce two-dimensional images of superficial blood flow [13]. Typically, a CCD camera collects the scattered light when a tissue is illuminated with a wide-field laser. This modality uses the laser speckle statistics. The camera integrates the fluctuating speckles during the exposure time and the image can be constructed after the speckle contrast is calculated. Due to the wide-field illumination, the penetration depth of this method is limited to less than about a millimeter. However, a relatively good lateral resolution is achieved. A somewhat higher depth resolution is obtained in the spatial frequency domain (e.g., with the use of structured illumination) [14,15].

A hybrid method with an increased signal-to-noise ratio known as the speckle contrast optical tomography (SCOT) has been recently proposed [16]. It is based on the use of massively parallel detectors such as CMOS cameras. In some implementations, SCOT can utilize the degrees of freedom in the data characteristic of the transport regime in addition to those typically used in the diffuse regime [17].

All methods described above are based on the use of the correlation diffusion equation for modeling the propagation of field correlations. It was shown experimentally [18] that the breakdown of the diffusion approximation in DCT experiments occurs in samples thinner than $\sim 3 \ell^{*}$, where $\ell^{*}$ is the transport mean free path. Theoretically, simulations showed that the diffusion approximation can be modified by accounting for the asymmetry parameter in order to be applicable down to few $\ell^{*}$ $[19,20]$. Indeed, the validity of the diffusion approximation depends on the level of anisotropy of the scatterers. It turns out that the diffusion approximation remains valid in smaller samples if they are highly anisotropic, as are some biological tissues such as muscles. As a result of the diffusion approximation, the reconstructed images have low spatial resolution [21]. Therefore, it is interesting to explore the transport regime. The mathematical model for propagation of correlations in this case is the CTE, which is similar but not equivalent to the radiative transport equation (RTE) that describes the intensity.

In this paper, we calculate the field correlation function in the transport regime as a solution to the CTE introduced in Refs. [5,22,23]. We show how perturbation theory can be applied to the CTE in order to calculate the sensitivity kernel relating the variation of the local Brownian motion of particles to the typical data. The Green's function of the standard radiative transport equation (RTE) can be used to construct the sensitivity kernel in the first Born approximation where the correlation time is considered to be the small parameter. We stress that the sensitivity kernel is defined for every point within the scattering medium. The sensitivity kernel is then the Jacobian matrix required in DCT in order to perform the image reconstruction [16]. Eventually, we demonstrate how the use of the CTE instead of the diffusion approximation can increase the contrast and resolution of reconstructions of dynamical properties of a scattering medium.

The paper is organized as follows: First, we develop the perturbation theory for the CTE and calculate the correlation sensitivity kernel in Sec. II. Then, we apply these results in order to evaluate the normalized field correlation function and the speckle contrast sensitivity in Sec. III. Examples of numerical evaluation of these quantities are shown in Sec. IV. A summary of the obtained results is given in Sec. V.

\section{PERTURBATIVE SOLUTION OF CORRELATION TRANSPORT EQUATION}

Two approaches can be chosen to treat the problem of multiple dynamic light scattering [6]. The first approach is to consider the sum of the "single" dynamic light scattering events over all allowed photon paths (i.e., the DWS). The second approach is based on the CTE. The second approach is the one we apply in this work due to its formal similarity with diffuse optical tomography.

We consider a multiply scattering medium with moving constituent particles, which can scatter light at the working wavelength. The statistics of laser speckles in the medium can be related to the field correlation function $\Gamma(\mathbf{r}, \hat{\mathbf{s}}, \tau)$. This function has been defined in Refs. [5,22,23] in terms of a Fourier transform of the two-point correlation function for the electric field (we use different notations: $\Gamma$ instead of $G_{1}$ with the symbol $G$ being reserved for the Green's function). What is important for us here is that $\Gamma$ can be related to measurements of the speckle contrast. Note that $\Gamma$ depends on the position $\mathbf{r}$, direction of propagation $\hat{\mathbf{s}}$, and the correlation time $\tau$. In a diffusion approximation-based approach, the directional degree of freedom $\hat{\mathbf{s}}$ is averaged out. We however do not disregard the dependence of $\Gamma$ on $\hat{\mathbf{s}}$. In the continuous-wave illumination regime, $\Gamma$ obeys the stationary $\mathrm{CTE}$

$$
\begin{aligned}
\left(\hat{\mathbf{s}} \cdot \nabla+\mu_{t}\right) \Gamma(\mathbf{r}, \hat{\mathbf{s}}, \tau)= & \mu_{s} \int \gamma^{s}\left(\mathbf{r}, \hat{\mathbf{s}}, \hat{\mathbf{s}}^{\prime}, \tau\right) A\left(\hat{\mathbf{s}}, \hat{\mathbf{s}}^{\prime}\right) \\
& \times \Gamma\left(\mathbf{r}, \hat{\mathbf{s}}^{\prime}, \tau\right) d^{2} s^{\prime}+\varepsilon(\mathbf{r}, \hat{\mathbf{s}}) .
\end{aligned}
$$

Here $\mu_{s}$ and $\mu_{t}$ are the scattering and extinction coefficients (assumed to be known), $A$ and $\varepsilon$ are the scattering phase function and the source term (the same functions that appear in the ordinary RTE), and $\gamma^{s}$ is the normalized temporal field correlation function for single scattering (defined in Ref. [5]) with the incoming and outgoing directions $\hat{\mathbf{s}}^{\prime}$ and $\hat{\mathbf{s}}$. Although the CTE (1) is stationary, it contains explicitly the correlation time $\tau$. Moreover, this equation is somewhat different from the ordinary RTE due to the presence of the function $\gamma^{s}$. The latter is given by [5]

$$
\gamma^{s}\left(\mathbf{r}, \hat{\mathbf{s}}, \hat{\mathbf{s}}^{\prime}, \tau\right)=\exp \left[-\frac{1}{3} k_{0}^{2}\left\langle\Delta^{2}(\mathbf{r}, \tau)\right\rangle\left(1-\hat{\mathbf{s}} \cdot \hat{\mathbf{s}}^{\prime}\right)\right]
$$

In this expression, $k_{0}$ is the wave number at the central frequency of the laser (assumed to be quasimonochromatic) and $\left\langle\Delta^{2}(\mathbf{r}, \tau)\right\rangle$ is the average squared drift of the moving particles initially located in the vicinity of the point $\mathbf{r}$ during the time $\tau$. Obviously, this mathematical description requires that $\Delta$ be much smaller than the characteristic scale on which the medium properties change. Equivalently, we can require the correlation time $\tau$ to be sufficiently small.

In the case of free Brownian motion of a particle in a liquid, we can use the well-known expression for the mean-square drift, viz, $\left\langle\Delta^{2}(\mathbf{r}, \tau)\right\rangle=6 D_{B}(\mathbf{r}) \tau$, where $D_{B}(\mathbf{r})$ is the diffusion coefficient for the moving particles (not to be confused with the diffusion coefficient for the energy density of light). Note that $D_{B}$ is a function of $\mathbf{r}$ since the medium properties can change. 
If $\tau$ is sufficiently small, we can expand $\gamma^{s}$ to first order as

$$
\gamma^{s}\left(\mathbf{r}, \hat{\mathbf{s}}, \hat{\mathbf{s}}^{\prime}, \tau\right) \approx 1+\left(\hat{\mathbf{s}} \cdot \hat{\mathbf{s}}^{\prime}-1\right) 2 D_{B}(\mathbf{r}) k_{0}^{2} \tau,
$$

and substituting the approximate expansion of $\gamma^{s}$ into (1) yields

$$
\begin{aligned}
(\hat{\mathbf{s}} \cdot \nabla & \left.+\mu_{t}\right) \Gamma(\mathbf{r}, \hat{\mathbf{s}}, \tau) \\
= & \mu_{s} \int A\left(\hat{\mathbf{s}}, \hat{\mathbf{s}}^{\prime}\right) \Gamma\left(\mathbf{r}, \hat{\mathbf{s}}^{\prime}, \tau\right) d^{2} s^{\prime} \\
& +\mu_{d}(\mathbf{r}, \tau) \int\left(\hat{\mathbf{s}} \cdot \hat{\mathbf{s}}^{\prime}-1\right) A\left(\hat{\mathbf{s}}, \hat{\mathbf{s}}^{\prime}\right) \Gamma\left(\mathbf{r}, \hat{\mathbf{s}}^{\prime}, \tau\right) d^{2} s^{\prime}+\varepsilon(\mathbf{r}, \hat{\mathbf{s}}) .
\end{aligned}
$$

We define the dynamic absorption coefficient as $\mu_{d}(\mathbf{r}, \tau)=$ $2 D_{B}(\mathbf{r}) k_{0}^{2} \mu_{s} \tau$. It plays the same role as the absorption coefficient in diffuse optical tomography (and has the same dimensionality of inverse length) but has a different physical origin. The field correlation function can be expanded in powers of $\tau$ as

$$
\Gamma(\mathbf{r}, \hat{\mathbf{s}}, \tau)=\Gamma_{0}(\mathbf{r}, \hat{\mathbf{s}})+\Gamma_{1}(\mathbf{r}, \hat{\mathbf{s}}) \tau+\Gamma_{2}(\mathbf{r}, \hat{\mathbf{s}}) \tau^{2}+\cdots .
$$

Upon substituting this expansion into (4) we find the equations for $\Gamma_{0}$ and $\Gamma_{1}$ :

$$
\begin{aligned}
& \left(\hat{\mathbf{s}} \cdot \nabla+\mu_{t}\right) \Gamma_{0}(\mathbf{r}, \hat{\mathbf{s}}) \\
& =\mu_{s} \int A\left(\hat{\mathbf{s}}, \hat{\mathbf{s}}^{\prime}\right) \Gamma_{0}\left(\mathbf{r}, \hat{\mathbf{s}}^{\prime}\right) d^{2} s^{\prime}+\varepsilon(\mathbf{r}, \hat{\mathbf{s}}), \\
& \left(\hat{\mathbf{s}} \cdot \nabla+\mu_{t}\right) \Gamma_{1}(\mathbf{r}, \hat{\mathbf{s}}) \\
& =\mu_{s} \int A\left(\hat{\mathbf{s}}, \hat{\mathbf{s}}^{\prime}\right) \Gamma_{1}\left(\mathbf{r}, \hat{\mathbf{s}}^{\prime}\right) d^{2} s^{\prime} \\
& +\frac{\mu_{d}(\mathbf{r}, \tau)}{\tau} \int\left(\hat{\mathbf{s}} \cdot \hat{\mathbf{s}}^{\prime}-1\right) A\left(\hat{\mathbf{s}}, \hat{\mathbf{s}}^{\prime}\right) \Gamma_{0}\left(\mathbf{r}, \hat{\mathbf{s}}^{\prime}\right) d^{2} s^{\prime} .
\end{aligned}
$$

It can be seen that $\Gamma_{0}$ is the solution to the ordinary RTE and is formally equal therefore to the specific intensity $I(\mathbf{r}, \widehat{\mathbf{s}})$. In particular, we can write

$$
\Gamma_{0}(\mathbf{r}, \hat{\mathbf{s}})=\int G\left(\mathbf{r}, \hat{\mathbf{s}} ; \mathbf{r}^{\prime}, \hat{\mathbf{s}}^{\prime}\right) \varepsilon\left(\mathbf{r}^{\prime}, \hat{\mathbf{s}}^{\prime}\right) d^{3} r^{\prime} d^{2} s^{\prime},
$$

where $G\left(\mathbf{r}, \hat{\mathbf{s}} ; \mathbf{r}^{\prime}, \hat{\mathbf{s}}^{\prime}\right)$ is the RTE Green's function for a homogeneous medium. Substituting this result into (6b), we can find $\Gamma_{1}$ :

$$
\begin{aligned}
\Gamma_{1}(\mathbf{r}, \hat{\mathbf{s}})= & \frac{1}{\tau} \int G\left(\mathbf{r}, \hat{\mathbf{s}} ; \mathbf{r}^{\prime}, \hat{\mathbf{s}}^{\prime}\right) \mu_{d}\left(\mathbf{r}^{\prime}, \tau\right)\left(\hat{\mathbf{s}}^{\prime} \cdot \hat{\mathbf{s}}^{\prime \prime}-1\right) A\left(\hat{\mathbf{s}}^{\prime}, \hat{\mathbf{s}}^{\prime \prime}\right) \\
& \times G\left(\mathbf{r}^{\prime}, \hat{\mathbf{s}}^{\prime \prime} ; \mathbf{r}^{\prime \prime}, \hat{\mathbf{s}}^{\prime \prime \prime}\right) \varepsilon\left(\mathbf{r}^{\prime \prime}, \hat{\mathbf{s}}^{\prime \prime \prime}\right) d^{3} r^{\prime} d^{2} s^{\prime} d^{2} s^{\prime \prime} d^{3} r^{\prime \prime} d^{2} s^{\prime \prime \prime} .
\end{aligned}
$$

Note that iterative evaluation of higher-order terms $\Gamma_{2}, \Gamma_{3}$, etc., is not mathematically justified because we have expanded $\gamma^{s}$ to first order in $\tau$ in (3). Evaluation of higher-order terms $\Gamma_{n}$ will require the use of higher-order expansion of $\gamma^{s}$. Thus the theory built here is restricted to first order in $\tau$.

Consider a point collimated source at $\mathbf{r}_{a}$ with the unique illumination direction $\hat{\mathbf{s}}_{a}$ so that $\varepsilon(\mathbf{r}, \widehat{\mathbf{s}})=\delta_{2}\left(\hat{\mathbf{s}}, \hat{\mathbf{s}}_{a}\right) \delta\left(\mathbf{r}-\mathbf{r}_{a}\right)$, where $\delta_{2}$ is the angular $\delta$ function. For the point of observation $\mathbf{r}_{b}$ and the direction of observation $\hat{\mathbf{s}}_{b}$, we define the data function as $\left.\tau \Gamma_{1}\left(\mathbf{r}_{b}, \hat{\mathbf{s}}_{b}\right)\right|_{\varepsilon=\delta\left(\mathbf{r}-\mathbf{r}_{a}\right) \delta_{2}\left(\hat{\mathbf{s}}, \hat{\mathbf{s}}_{a}\right)}=\Phi$ so that we can write

$$
\Phi\left(\mathbf{r}_{b}, \hat{\mathbf{s}}_{b} ; \mathbf{r}_{a}, \hat{\mathbf{s}}_{a}, \tau\right)=\int K_{1}\left(\mathbf{r}_{b}, \hat{\mathbf{s}}_{b} ; \mathbf{r}_{a}, \hat{\mathbf{s}}_{a} ; \mathbf{r}\right) \mu_{d}(\mathbf{r}, \tau) d^{3} r,
$$

where

$$
\begin{aligned}
K_{1}\left(\mathbf{r}_{b}, \hat{\mathbf{s}}_{b} ; \mathbf{r}_{a}, \hat{\mathbf{s}}_{a} ; \mathbf{r}\right)= & \int G\left(\mathbf{r}_{b}, \hat{\mathbf{s}}_{b} ; \mathbf{r}, \hat{\mathbf{s}}\right)\left(\hat{\mathbf{s}} \cdot \hat{\mathbf{s}}^{\prime}-1\right) \\
& \times A\left(\hat{\mathbf{s}}, \hat{\mathbf{s}}^{\prime}\right) G\left(\mathbf{r}, \hat{\mathbf{s}}^{\prime} ; \mathbf{r}_{a}, \hat{\mathbf{s}}_{a}\right) d^{2} s d^{2} s^{\prime},
\end{aligned}
$$

is the sensitivity kernel of DCT. It quantifies the variations of the field correlation function due to the inhomogeneities of the diffusion coefficient $D_{B}(\mathbf{r})$. We emphasize that $\Gamma_{1}(\mathbf{r}, \hat{\mathbf{s}})$ can be computed if the correlation time-dependent function $\Gamma\left(\mathbf{r}_{b}, \hat{\mathbf{s}}, \tau\right)$ is known for some range of $\tau$ by using linear regression.

It is important for us that the sensitivity kernel $K_{1}$ defined in (10) is expressed in terms of the Green's function of the ordinary RTE. The kernel is similar to the sensitivity kernel of diffuse optical tomography for the absorptive contrast [24] but contains an extra factor $\left(\hat{\mathbf{s}} \cdot \hat{\mathbf{s}}^{\prime}-1\right) A\left(\hat{\mathbf{s}}, \hat{\mathbf{s}}^{\prime}\right)$; in the theory of Ref. [24], this factor is replaced by the angular $\delta$ function $\delta_{2}\left(\hat{\mathbf{s}}, \hat{\mathbf{s}}^{\prime}\right)$. In spite of some mathematical similarities, the two kernels describe sensitivity to very different contrast mechanisms. The kernel of diffuse optical tomography quantifies the sensitivity to absorbing chromophores while the kernel derived here quantifies sensitivity to local mobility of the particles comprising the medium.

\section{APPLICATION TO SPECKLE CONTRAST OPTICAL TOMOGRAPHY}

In the following we show how the correlation sensitivity kernel of DCT can be used to calculate quantities useful in SCOT.

In SCOT [16], the reconstruction of the distribution of the mean-square displacement $\left\langle\Delta r^{2}(\mathbf{r}, \tau)\right\rangle$ into the sample volume is recovered from measurements at the surface of the speckle contrast sensitivity. The speckle contrast is defined as $[16,21]$

$$
\sigma^{2}\left(\mathbf{r}_{b}, \hat{\mathbf{s}}_{b}, T\right)=\frac{2 \beta}{T} \int_{0}^{T}\left|\gamma\left(\mathbf{r}_{b}, \hat{\mathbf{s}}_{b}, \tau\right)\right|^{2}\left(1-\frac{\tau}{T}\right) d \tau,
$$

where $\beta$ is an experimental parameter depending on the number of speckles and the detection conditions. The term $T$ is the exposure time of the CCD camera collecting the scattered radiation and in order to apply the linearization of (3) it is required that $T<400 \mu \mathrm{s}$. The measurable quantity is the normalized correlation function $[8,25]$

$$
\gamma\left(\mathbf{r}_{b}, \hat{\mathbf{s}}_{b}, \tau\right)=\frac{\Gamma\left(\mathbf{r}_{b}, \hat{\mathbf{s}}_{b}, \tau\right)}{I\left(\mathbf{r}_{b}, \hat{\mathbf{s}}_{b}\right)} .
$$

Here $I\left(\mathbf{r}_{b}, \hat{\mathbf{s}}_{b}\right)$ is the specific intensity at the detector, which is characterized by the position $\mathbf{r}_{b}$ and collimation direction $\hat{\mathbf{s}}_{b}$. For sufficiently small correlation times, we can use (5) to approximate the right-hand side of (12) to first order in $\tau$. Recalling that the expansion coefficient $\Gamma_{0}$ in (5) is, in fact, the specific intensity, we arrive at

$$
\gamma\left(\mathbf{r}_{b}, \hat{\mathbf{s}}_{b}, \tau\right)=1+\frac{\Gamma_{1}\left(\mathbf{r}_{b}, \hat{\mathbf{s}}_{b}\right)}{\Gamma_{0}\left(\mathbf{r}_{b}, \hat{\mathbf{s}}_{b}\right)} \tau .
$$


Substituting (13) into (11) yields

$$
\sigma^{2}\left(\mathbf{r}_{b}, \hat{\mathbf{s}}_{b}, T\right)=\frac{2 \beta}{T} \int_{0}^{T}\left|1+\frac{\Gamma_{1}\left(\mathbf{r}_{b}, \hat{\mathbf{s}}_{b}\right)}{\Gamma_{0}\left(\mathbf{r}_{b}, \hat{\mathbf{s}}_{b}\right)} \tau\right|^{2}\left(1-\frac{\tau}{T}\right) d \tau .
$$

For a point collimated source characterized by position $\mathbf{r}_{a}$ and direction $\hat{\mathbf{s}}_{a}$, keeping just the terms at first order in $\tau$, we can define the SCOT data function according to

$$
\Phi_{\mathrm{SCOT}}\left(\mathbf{r}_{b}, \hat{\mathbf{s}}_{b} ; \mathbf{r}_{a}, \hat{\mathbf{s}}_{a}, T\right)=\sigma^{2}\left(\mathbf{r}_{b}, \hat{\mathbf{s}}_{b}, T\right)-\beta,
$$

and it follows that

$$
\Phi_{\mathrm{SCOT}}\left(\mathbf{r}_{b}, \hat{\mathbf{s}}_{b} ; \mathbf{r}_{a}, \hat{\mathbf{s}}_{a}, T\right)=\frac{4 \beta}{T} \frac{\Gamma_{1}\left(\mathbf{r}_{b}, \hat{\mathbf{s}}_{b}\right)}{\Gamma_{0}\left(\mathbf{r}_{b}, \hat{\mathbf{s}}_{b}\right)} \int_{0}^{T}\left(\tau-\frac{\tau^{2}}{T}\right) d \tau .
$$

Finally, it can be seen that this data function is coupled through $\mu_{d}(\mathbf{r}, T)$ by the linear integral equation

$$
\begin{aligned}
\Phi_{\text {SCOT }} & =\frac{2}{3} \beta \int \frac{K_{1}\left(\mathbf{r}_{b}, \hat{\mathbf{s}}_{b} ; \mathbf{r}_{a}, \hat{\mathbf{s}}_{a} ; \mathbf{r}\right)}{\Gamma_{0}\left(\mathbf{r}_{b}, \hat{\mathbf{s}}_{b}\right)} \mu_{d}(\mathbf{r}, T) d^{3} r \\
& =\frac{2 \beta}{3 \Gamma_{0}\left(\mathbf{r}_{b}, \hat{\mathbf{s}}_{b}\right)} \Phi .
\end{aligned}
$$

Therefore, to first order in $\tau$, the sensitivity kernel of SCOT is the same as the sensitivity kernel of DCT normalized to the local intensity and multiplied by $\frac{2}{3} \beta$. This kernel can be calculated with (10). Here $\Phi_{\text {SCOT }}$ is expressed in terms of experimentally measurable quantities as $\sigma^{2}$ and $\beta$. Correspondingly, (17) can be inverted by standard methods of linear algebra to find the unknown contrast $\mu_{d}(\mathbf{r}, T)$. The difference between SCOT and DCT is that different measurements must be performed and then differently postprocessed to obtain the data function $\Phi$. Note that linearization of the correlation function $\Gamma$ in $\tau$, which was used to arrive at the definition (9) of the data function, is valid in typical experiments for $\tau \leqslant 400 \mu$ s assuming $D_{B} \sim 0.5 \times 10^{-8} \mathrm{~cm}^{2} \mathrm{~s}^{-1}$ and for the laser frequency in the visible spectral range.

Moreover, it is necessary to stress the importance of writing the sensitivity through kernels since the local dependence on position inside the medium is maintained (equivalently to DCT Jacobians while, the sensitivity as defined in this paper depends on the source and detector positions only). In addition, (17) takes into account the variation with respect to a static medium, i.e., satisfying the RTE. Thus, the sensitivity can be calculated by summing only onto the dynamic portion of the medium (i.e., the perturbation) and no modification of (11) is necessary in order to correct for scattering from the static part (as proposed in Ref. [21]).

In what follows we show results for the temporal field correlation sensitivity kernel which are relevant for DCT in the correlation transport regime.

\section{RESULTS}

We now consider the imaging of a scattering medium in the shape of a slab. The scattering and absorption coefficients $\left(\mu_{s}=10 \mathrm{~cm}^{-1}\right.$ and $\left.\mu_{a}=0.02 \mathrm{~cm}^{-1}\right)$, and the scattering phase function (for Mie particles with asymmetry parameter $g=0.95$ and size parameter $x=7.15)$, which corresponds to a transport mean free path $\ell^{*}=1 /\left[\mu_{a}+(1-g) \mu_{s}\right]=$
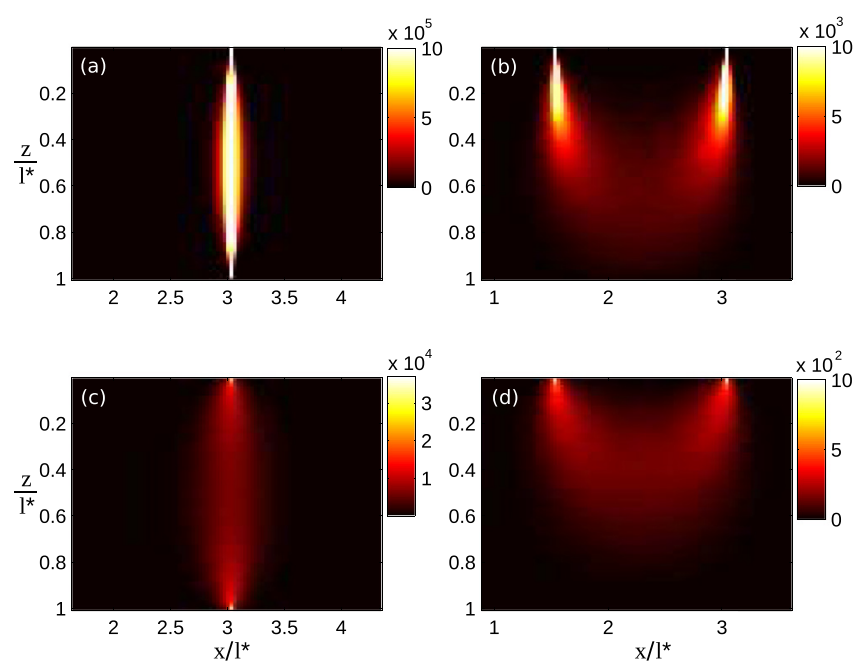

FIG. 1. Correlation function sensitivity kernel computed via Monte Carlo simulations in (a), (c) transmission geometry and (b), (d) reflection geometry. The solution of the CTE is shown in panels (a) and (b) and its $P_{1}$ approximation in panels (c) and (d). All distances are shown in units of $\ell^{*}$. The source-detector separation in panels (b) and $(\mathrm{d})$ is $1.5 \ell^{*}$.

$1.92 \mathrm{~cm}$ are constant inside the medium but the scatterer Brownian motion diffusion coefficient $D_{B}$ can vary in space. The propagation of the field temporal correlation is assumed to obey the CTE. The source is a continuous-wave, collimated laser beam incident at some location $\mathbf{r}_{A}$ on the surface of the slab and in the direction of the unit vector $\hat{\mathbf{s}}_{A}$.

Since the temporal field correlation function cannot be measured directly, the detector measures the intensity fluctuations within a single speckle area centered at a point $\mathbf{r}_{B}$ and in the direction $\hat{\mathbf{s}}_{B}$. A correlator should be used to get the normalized temporal intensity correlation function [12] and, under the assumption of Gaussian statistics of the field fluctuations, the Siegert relationship can then be used to convert it into the normalized electric-field autocorrelation. Then, the presence of heterogeneities of the mean-squared displacement inside the slab will result in a detectable variation of the measured field correlation function due to the variations in the dynamic absorption. Within the accuracy of the first Born approximation, the equation that relates the fluctuations of the Brownian motion diffusion coefficient $D_{B}$ to the sensitivity function is expressed through (9).

First, simulations relevant for DCT are presented in the form of sensitivity to dynamic absorption. The field correlation sensitivity kernel $K_{1}(10)$ is illustrated in Fig. 1. To compute $K_{1}$, we use Monte Carlo simulations solving numerically the RTE (and thus find the Green's function $G$ ). The coordinates are in unit of $\ell^{*}$, the transport mean free path. The Green's function is calculated with $N=10^{8}$ number of photons for a slab of thickness $L=1 \ell^{*}$. In addition, the reciprocity relation [26] is applied to the Green's function propagating to the detector (although here we do not consider polarization). Then, an expansion in spherical harmonics of all angularly dependent functions is used; details of angular integration of (10) are explained in Appendix A. The approximated solution to the RTE is evaluated through the $P_{1}$ approximation. Strictly 

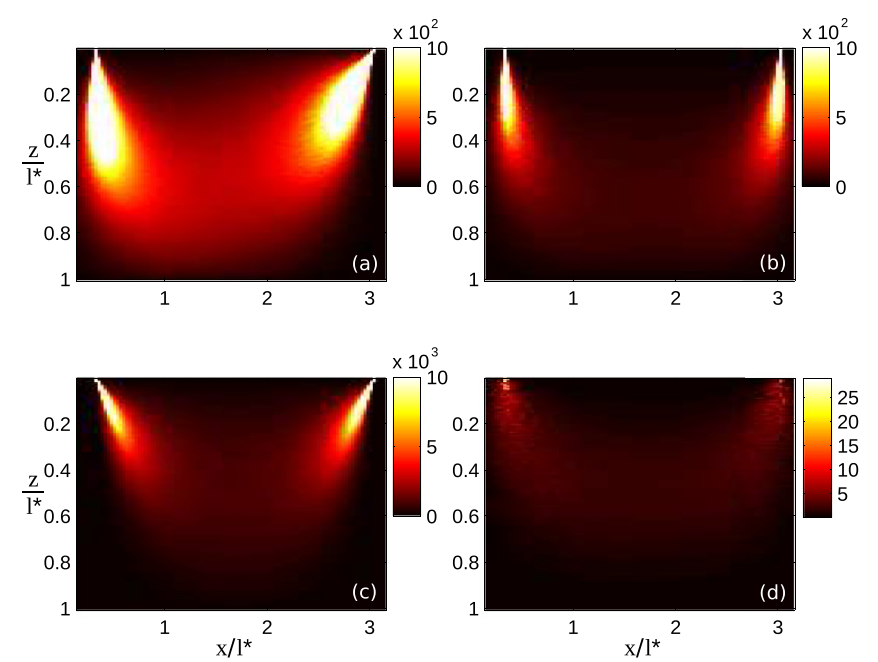

FIG. 2. Correlation function sensitivity kernel computed via Monte Carlo simulations in reflection geometry. The solution of the CTE is shown in panels (a)-(c) and its $P_{1}$ approximation in panel (d). The source-detector separation is $2.7 \ell^{*}$. In panels (a) and (c) the source and detector are rotated.

speaking, the $P_{1}$ approximation can be in general more accurate than the diffusion approximation. Consequently, the results can be extrapolated to the diffusion approximation also.

The left and right columns of images in Fig. 1 show the results obtained in the transmission and the reflection geometries, respectively. The top row shows $K_{1}$ obtained by solving the CTE rigorously as described above and the bottom row was obtained by using the $P_{1}$ approximation wherein the spherical function expansion of the phase function is truncated at $l=1$ and the correlation diffusion kernel is truncated at $l=0$ since, for $\tau \ll\left(2 D k_{0}^{2}\right)^{-1}$, the $l=1$ term goes rapidly to zero. In addition, the correlation sensitivity kernel in the $P_{1}$ approximation is calculated for an isotropic source. In contrast, the CTE solutions are obtained considering up to $l=15$ terms in the spherical harmonic expansion of the sensitivity kernel. As expected, increasing the source-detector distance leads to a larger sensitivity to deep regions.

In Fig. 2, we show that using the CTE allows one to exploit some additional degrees of freedom in the data, which are lost in the $P_{1}$ approximation, i.e., the source-detector orientation. It is possible to rotate the source-detector system in a way to maximize the signal from a specific area of the sample or even to scan in depth the sample without changing the sourcedetector distance.

It is also appreciable that, both in transmission and reflection geometries, the volume sampled using the CTE solution is much smaller than in the case when the $P_{1}$ approximation is used. As a consequence, if a scanning strategy is adopted (see the setup in Fig. 3), an increased resolution of dynamically absorbing objects is obtained within the transport regime. This is demonstrated in Fig. 4. To obtain these results, it is necessary to scan the sample with fixed relative position of the source and detector (both in the transmission and reflection geometries the source and detector are aligned). See Ref. [27] for more details about the direct imaging procedure. Then, a dynamically absorbing object in the shape of a straight line is

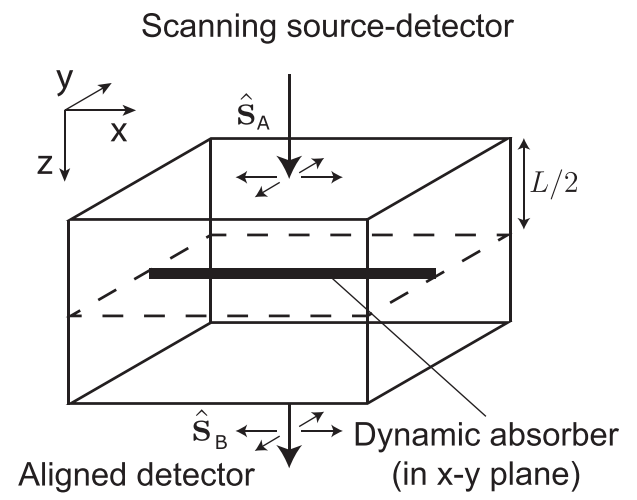

FIG. 3. Geometry used in simulated experiment for imaging a dynamic absorber (straight capillary). The source and detector have normal orientation with respect to the surface.

considered (see again Fig. 3). This simulates the presence in the sample of a region with particles (red blood cells) undergoing Brownian motion and can be thought of as a straight capillary in a tissue. The correlation sensitivity kernel needs to be summed over the volume occupied by the object in order to calculate the sensitivity at the detector [i.e., evaluating (9)]. Scanning on the sample surface, the sensitivity can be visualized as an image displaying the shadow of the dynamic absorbing object. It is interesting to note the higher resolution obtained using the CTE while, in the $P_{1}$ approximation the image is blurred due to long path photons. In addition, within this scanning strategy, the transmission geometry seems to provide better resolution. All the results were calculated for the same Mie particles as the previous examples. The slab thickness in this experiment
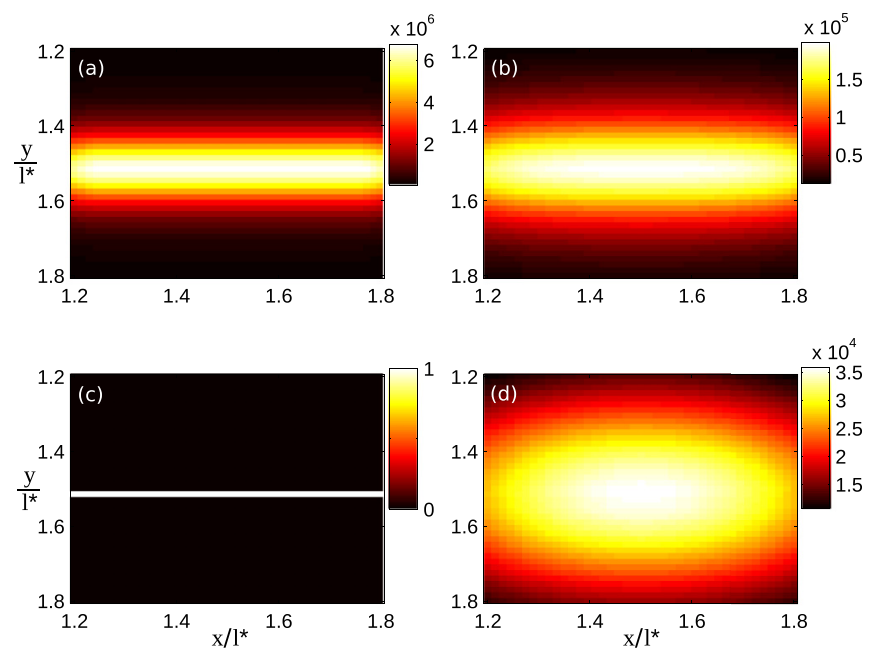

FIG. 4. Correlation function sensitivity computed via Monte Carlo simulations. The images are obtained by scanning on the surface of the sample containing a tube filled with particles undergoing Brownian motion with $D_{B}=0.5 \times 10^{-8} \mathrm{~cm}^{2} \mathrm{~s}^{-1}$. The images correspond to the shadow of the dynamically absorbing object (straight capillary) shown in panel (c). The solution of the CTE is shown in panels (a) and (b) for transmission and reflection geometries respectively. The result for the $P_{1}$ approximation is shown in panel (d) in transmission geometry (very similar result is obtained in reflection geometry). The slab thickness is $1 \ell^{*}$. 

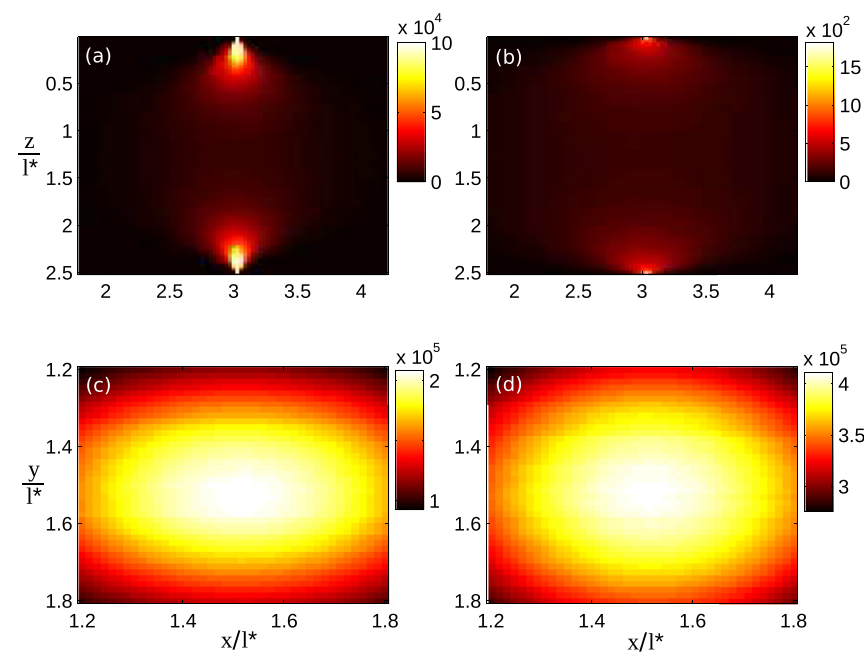

FIG. 5. (a), (b) Correlation function sensitivity kernel in transmission geometry. (c), (d) Correlation function sensitivity, where the images correspond to the shadow of the dynamically absorbing object. (a), (c) The CTE solution and (b), (d) its $P_{1}$ approximation. The slab thickness is $2.5 \ell^{*}$.

was set to $1 \ell^{*}$ thus constraining the photons into the transport regime. The diffuse propagation regime can be achieved by increasing the slab thickness thus forcing the photons to travel over longer paths before arriving at the detector. The transition to the diffuse propagation regime is presented in Fig. 5. It is then clear that for samples thicker than around $2.5 \ell^{*}$ the photons start to travel along trajectories that are far from the source-detector axis. Hence, image blurring is appearing due to the broad distribution of photons presented in the first row of Fig. 5. The solution of the CTE tends to the $P_{1}$ approximation at a scale that is consistent with the results of Ref. [18] (in transmission geometry for high anisotropic media $g=0.95$ ).
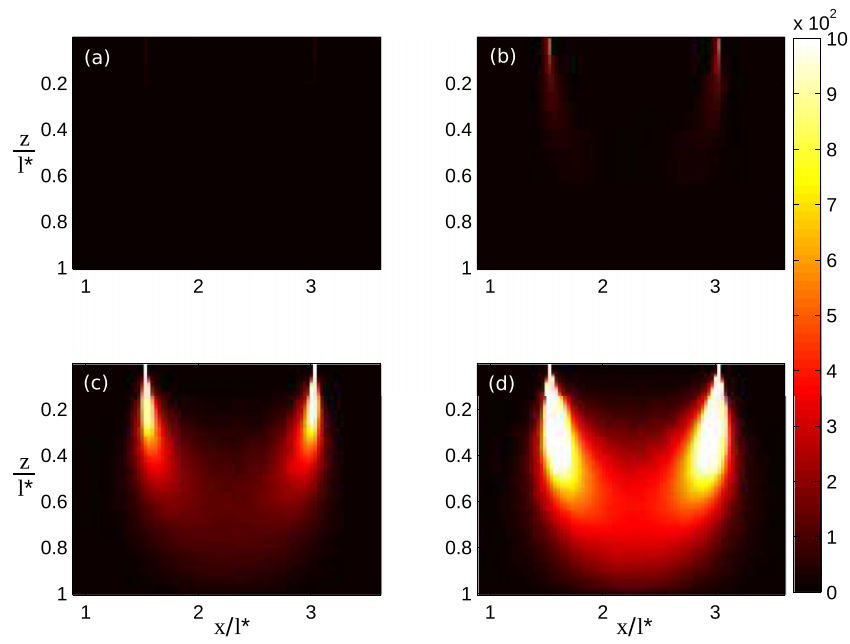

FIG. 6. Correlation function sensitivity kernel computed via Monte Carlo simulations in reflection geometry solving the CTE with dynamic absorption corresponding to different correlation times (a) $\tau=1 \mu \mathrm{s}$, (b) $\tau=10 \mu \mathrm{s}$, (c) $\tau=100 \mu \mathrm{s}$, and (d) $\tau=300 \mu \mathrm{s}$. The source-detector separation is $1.5 \ell^{*}$.
Finally, Fig. 6 shows the sensitivity kernel to dynamic absorption in reflection geometry at different correlation times ( $\tau=1,10,100,300 \mu \mathrm{s}$ ). These results are relevant for SCOT demonstrating sensitivity to different regions depending on the timescale of the experiment (the CCD camera exposure time $T$ ). As the correlation time increases, the measurement becomes more and more sensitive to the region between the source and detector. In addition, the volume around the source and detector gets a very high sensitivity which can lead to the suppression of the signal coming from the interstitial region. Experimentally, a method should be designed in order to minimize the contribution of sample volumes around the source and detector. A fast solution can be the subtraction from the measurement at late times of the data at early times in a way to cancel the high-intensity contributions of the source and detector.

\section{SUMMARY}

An analytical expression for the temporal field correlation function sensitivity in the transport regime is derived by perturbative analysis of the CTE. Its kernel is the Jacobian required in DCT in order to perform image reconstruction and it is also directly relevant to SCOT. The DCT kernel is evaluated numerically in order to demonstrate the higher resolution and contrast that can be obtained when operating DCT imaging in the transport regime (source-detector separation lower than approximately $2 \mathrm{~cm}$ for the medium considered here).

Furthermore, we investigated the region of transition between the correlation transport regime and the diffuse propagation regime. For the high scattering anisotropy of the medium ( $g=0.95$ ), the $P_{1}$ approximation is expected to yield accurate results already for a source-detector separation of $\sim 2 \ell^{*}$. However, at smaller propagation distances, the solution to the CTE captures the angular dependence of the correlation function exactly while, the $P_{1}$ approximation distorts significantly the single-scattering angular distribution. One can say that, in the $P_{1}$ approximation, as well as in the more general family of diffusion approximations [28], the only trace of the phase function is the scattering asymmetry parameter $g$. However, this parameter does not characterize the scattering process completely, and at small propagation distances, different phase functions with the same values of $g$ can produce significantly different results. The use of CTE provides a systematic way to account for this effect.

An inaccurate representation of the scattering phase function in the $P_{1}$ approximation has the consequence of predicting longer photon paths that, in turn, cause blurring of the image of the capillary. For the simulated imaging procedure based on scanning with an on-axis source-detector pair, the photon path information is relevant. Hence, it is advantageous in the transport regime to use the CTE solution as this can yield substantially improved images. This is in agreement with the results obtained in Ref. [10]. As expected, if the source-detector separation is $\geqslant 3 \ell^{*}$, the two approaches yield essentially the same result (for the medium considered here).

To conclude, the application of our method to DCT allows one to image arbitrary-shaped regions filled with particles undergoing Brownian motion. Moreover, arbitrary sourcedetector separations and orientations can be evaluated with a 
single simulation, providing additional degrees of freedom in the data. This is important for DCT when many source-detector positions are employed. Concerning SCOT, it is demonstrated how the sensitivity changes increasing the exposure time of the CCD camera. We expect that the above results will be useful for DCT and SCOT and will facilitate the development of efficient and accurate reconstruction methods for highresolution tomographic imaging of the dynamical properties of multiply scattering media.

\section{ACKNOWLEDGMENTS}

This work was supported by the A*MIDEX Project, grant ANR-11-IDEX-0001-02, funded by the "Investissements d'Avenir" French Government program, managed by the Agence Nationale de la Recherche (ANR).

\section{APPENDIX: ANGULAR INTEGRATION}

We first make use of the reciprocity relation for the Green's function as introduced in Ref. [26] (although here we do not consider polarization). The sensitivity kernel can then be rewritten as

$$
\begin{aligned}
& K_{1}\left(\mathbf{r}_{b}, \hat{\mathbf{s}}_{b} ; \mathbf{r}_{a}, \hat{\mathbf{s}}_{a} ; \mathbf{r}^{\prime}\right)=\int G\left(\mathbf{r}_{2},-\hat{\mathbf{s}}^{\prime} ; \mathbf{r}_{a}, \hat{\mathbf{s}}_{a}\right) \\
& \quad \times\left(\hat{\mathbf{s}}^{\prime} \cdot \hat{\mathbf{s}}^{\prime \prime}-1\right) A\left(\hat{\mathbf{s}}^{\prime}, \hat{\mathbf{s}}^{\prime \prime}\right) G\left(\mathbf{r}^{\prime}, \hat{\mathbf{s}}^{\prime \prime} ; \mathbf{r}_{a}, \hat{\mathbf{s}}_{a}\right) d^{2} s^{\prime} d^{2} s^{\prime \prime},
\end{aligned}
$$

where using the translational invariance for a homogeneous sample gives $\mathbf{r}_{2}=\mathbf{r}^{\prime}+\mathbf{r}_{a}-\mathbf{r}_{b}$.

As noted in Ref. [5], the $P_{N}$ approximation can be applied to the correlation transport equation (1) with the additional expansion in spherical harmonics, $Y_{l m}$, of the normalized temporal field correlation function for single scattering $\gamma^{s}$. Following this approach, we consider the expansion of the sensitivity kernel as introduced in (A1). The unperturbed Green's function solving the RTE reads

$$
G\left(\mathbf{r}^{\prime}, \hat{\mathbf{s}}^{\prime \prime} ; \mathbf{r}_{a}, \hat{\mathbf{s}}_{a}\right)=\sum_{l=0}^{l_{\max }} \sum_{m=-l}^{l} a_{l m}\left(\mathbf{r}^{\prime}\right) Y_{l m}\left(\hat{\mathbf{s}}^{\prime \prime}\right) .
$$

The phase function truncated expansion (up to the maximum angular-momentum order $l_{\max }$ ) is

$$
A\left(\hat{\mathbf{s}}^{\prime}, \hat{\mathbf{s}}^{\prime \prime}\right)=\sum_{l=0}^{l_{\max }} \sum_{m=-l}^{l} g_{l} Y_{l m}^{*}\left(\hat{\mathbf{s}}^{\prime}\right) Y_{l m}\left(\hat{\mathbf{s}}^{\prime \prime}\right)
$$

where $g_{l}$ are the phase function moments. Lastly, the perturbation of the phase function caused by $\gamma^{s}$ reads

$$
\hat{\mathbf{s}}^{\prime} \cdot \hat{\mathbf{s}}^{\prime \prime}-1=-1+\frac{4 \pi}{3} \sum_{m=-1}^{1} Y_{1 m}^{*}\left(\hat{\mathbf{s}}^{\prime}\right) Y_{1 m}\left(\hat{\mathbf{s}}^{\prime \prime}\right) .
$$

Hence, the sensitivity kernel can be rewritten as

$$
\begin{aligned}
& K_{1}\left(\mathbf{r}_{b}, \hat{\mathbf{s}}_{b} ; \mathbf{r}_{a}, \hat{\mathbf{s}}_{a} ; \mathbf{r}^{\prime}\right) \\
& =\int \sum_{l=0}^{l_{\max }} \sum_{m=-l}^{l}(-1)^{l} a_{l m}\left(\mathbf{r}_{2}\right) Y_{l m}\left(\hat{\mathbf{s}}^{\prime}\right)
\end{aligned}
$$

$$
\begin{aligned}
& \times \sum_{l^{\prime}=0}^{l_{\max }} \sum_{m^{\prime}=-l^{\prime}}^{l^{\prime}} g_{l^{\prime}} Y_{l^{\prime} m^{\prime}}^{*}\left(\hat{\mathbf{s}}^{\prime}\right) Y_{l^{\prime} m^{\prime}}\left(\hat{\mathbf{s}}^{\prime \prime}\right) \\
& \times\left[-1+\frac{4 \pi}{3} \sum_{m^{\prime \prime}=-1}^{1} Y_{1 m^{\prime \prime}}^{*}\left(\hat{\mathbf{s}}^{\prime}\right) Y_{1 m^{\prime \prime}}\left(\hat{\mathbf{s}}^{\prime \prime}\right)\right] \\
& \times \sum_{l^{\prime \prime \prime}=0}^{l_{\max }} \sum_{m^{\prime \prime \prime}=-l^{\prime \prime \prime}}^{l^{\prime \prime \prime}} a_{l^{\prime \prime \prime} m^{\prime \prime \prime}}\left(\mathbf{r}^{\prime}\right) Y_{l^{\prime \prime \prime} m^{\prime \prime \prime}}\left(\hat{\mathbf{s}}^{\prime \prime}\right) d^{2} s^{\prime} d^{2} s^{\prime \prime},
\end{aligned}
$$

where we made use of the relation $Y_{l m}(-\hat{\mathbf{s}})=(-1)^{l} Y_{l m}(\hat{\mathbf{s}})$ for the inversion of direction. Then, considering only the first term into the square brackets and doing the integrals, exploiting the orthogonality of the spherical harmonics, we get

$$
-\sum_{l=0}^{l_{\max }} \sum_{m=-l}^{l} g_{l}(-1)^{l} a_{l m}\left(\mathbf{r}_{2}\right) a_{l m}\left(\mathbf{r}^{\prime}\right) .
$$

The second term in the square brackets is more complicated. This needs to be calculated considering the following products:

$$
\begin{aligned}
Y_{l m}(\hat{\mathbf{s}}) Y_{1-1}(\hat{\mathbf{s}})= & \sqrt{\frac{3}{8 \pi}} B_{l+1}^{m-1} Y_{l+1 m-1}(\hat{\mathbf{s}}) \\
& -\sqrt{\frac{3}{8 \pi}} B_{l}^{-m} Y_{l-1 m-1}(\hat{\mathbf{s}}), \\
Y_{l m}(\hat{\mathbf{s}}) Y_{10}(\hat{\mathbf{s}})= & \sqrt{\frac{3}{4 \pi}} A_{l+1}^{m} Y_{l+1 m}(\hat{\mathbf{s}}) \\
& +\sqrt{\frac{3}{4 \pi}} A_{l}^{m} Y_{l-1 m}(\hat{\mathbf{s}}), \\
Y_{l m}(\hat{\mathbf{s}}) Y_{11}(\hat{\mathbf{s}})= & \sqrt{\frac{3}{8 \pi}} B_{l+1}^{-m-1} Y_{l+1 m+1}(\hat{\mathbf{s}}) \\
& -\sqrt{\frac{3}{8 \pi}} B_{l}^{m} Y_{l-1 m+1}(\hat{\mathbf{s}}),
\end{aligned}
$$

where the coefficients $A_{l}^{m}$ and $B_{l}^{m}$ are special cases of the $3 j$ symbols

$$
\begin{aligned}
& A_{l}^{m}=\left[\frac{(l-m)(l+m)}{(2 l-1)(2 l+1)}\right]^{\frac{1}{2}}, \\
& B_{l}^{m}=\left[\frac{(l-m)(l-m-1)}{(2 l-1)(2 l+1)}\right]^{\frac{1}{2}} .
\end{aligned}
$$

Thus, we can simplify the term (see the appendix in Ref. [5])

$$
\begin{aligned}
& \frac{4 \pi}{3} \sum_{l^{\prime}=0}^{l_{\max }} \sum_{m^{\prime}=-l^{\prime}}^{l^{\prime}} g_{l^{\prime}} Y_{l^{\prime} m^{\prime}}^{*}\left(\hat{\mathbf{s}}^{\prime}\right) Y_{l^{\prime} m^{\prime}}\left(\hat{\mathbf{s}}^{\prime \prime}\right) \sum_{m^{\prime \prime}=-1}^{1} Y_{1 m^{\prime \prime}}^{*}\left(\hat{\mathbf{s}}^{\prime}\right) Y_{1 m^{\prime \prime}}\left(\hat{\mathbf{s}}^{\prime \prime}\right) \\
& =\frac{4 \pi}{3} \sum_{l^{\prime}=0}^{l_{\max }} \sum_{m^{\prime}=-l^{\prime}}^{l^{\prime}} g_{l^{\prime}} \\
& \quad \times\left\{\frac{3}{8 \pi}\left[B_{l^{\prime}+1}^{m^{\prime}-1} Y_{l^{\prime}+1 m^{\prime}-1}^{*}\left(\hat{\mathbf{s}}^{\prime}\right)-B_{l^{\prime}}^{-m^{\prime}} Y_{l^{\prime}-1 m^{\prime}-1}^{*}\left(\hat{\mathbf{s}}^{\prime}\right)\right]\right. \\
& \quad \times\left[B_{l^{\prime}+1}^{m^{\prime}-1} Y_{l^{\prime}+1 m^{\prime}-1}\left(\hat{\mathbf{s}}^{\prime \prime}\right)-B_{l^{\prime}}^{-m^{\prime}} Y_{l^{\prime}-1 m^{\prime}-1}\left(\hat{\mathbf{s}}^{\prime \prime}\right)\right]
\end{aligned}
$$




$$
\begin{aligned}
& +\frac{3}{4 \pi}\left[A_{l^{\prime}+1}^{m^{\prime}} Y_{l^{\prime}+1 m^{\prime}}^{*}\left(\hat{\mathbf{s}}^{\prime}\right)+A_{l^{\prime}}^{m^{\prime}} Y_{l^{\prime}-1 m^{\prime}}^{*}\left(\hat{\mathbf{s}}^{\prime}\right)\right] \\
& \times\left[A_{l^{\prime}+1}^{m^{\prime}} Y_{l^{\prime}+1 m^{\prime}}\left(\hat{\mathbf{s}}^{\prime \prime}\right)+A_{l^{\prime}}^{m^{\prime}} Y_{l^{\prime}-1 m^{\prime}}\left(\hat{\mathbf{s}}^{\prime \prime}\right)\right] \\
& -\frac{3}{8 \pi}\left[B_{l^{\prime}+1}^{-m^{\prime}-1} Y_{l^{\prime}+1 m^{\prime}+1}^{*}\left(\hat{\mathbf{s}}^{\prime}\right)-B_{l^{\prime}}^{m^{\prime}} Y_{l^{\prime}-1 m^{\prime}+1}^{*}\left(\hat{\mathbf{s}}^{\prime}\right)\right] \\
& \left.\times\left[B_{l^{\prime}+1}^{-m^{\prime}-1} Y_{l^{\prime}+1 m^{\prime}+1}\left(\hat{\mathbf{s}}^{\prime \prime}\right)-B_{l^{\prime}}^{m^{\prime}} Y_{l^{\prime}-1 m^{\prime}+1}\left(\hat{\mathbf{s}}^{\prime \prime}\right)\right]\right\} .
\end{aligned}
$$

Furthermore, we can proceed evaluating the integral over $\hat{\mathbf{s}}^{\prime}$

$$
\begin{aligned}
\int \sum_{l=0}^{l_{\max }} \sum_{m=-l}^{l}(-1)^{l} a_{l m}\left(\mathbf{r}_{2}\right) Y_{l m}\left(\hat{\mathbf{s}}^{\prime}\right) \\
\quad \times \frac{4 \pi}{3} \sum_{l^{\prime}=0}^{l_{\max }} \sum_{m^{\prime}=-l^{\prime}}^{l^{\prime}} g_{l^{\prime}} Y_{l^{\prime} m^{\prime}}^{*}\left(\hat{\mathbf{s}}^{\prime}\right) Y_{l^{\prime} m^{\prime}}\left(\hat{\mathbf{s}}^{\prime \prime}\right) \\
\quad \times \sum_{m^{\prime \prime}=-1}^{1} Y_{1 m^{\prime \prime}}^{*}\left(\hat{\mathbf{s}}^{\prime}\right) Y_{1 m^{\prime \prime}}\left(\hat{\mathbf{s}}^{\prime \prime}\right) d^{2} s^{\prime} \\
=\frac{4 \pi}{3} \sum_{l=0}^{l_{\max }} \sum_{m=-l}^{l}(-1)^{l} a_{l m}\left(\mathbf{r}_{2}\right) \\
\quad \times\left\{\frac{3}{8 \pi} g_{l-1} B_{l}^{m}\left[B_{l}^{m} Y_{l m}\left(\hat{\mathbf{s}}^{\prime \prime}\right)-B_{l-1}^{-m-1} Y_{l-2 m}\left(\hat{\mathbf{s}}^{\prime \prime}\right)\right]\right. \\
\quad-\frac{3}{8 \pi} g_{l+1} B_{l+1}^{-m-1}\left[B_{l+2}^{m} Y_{l+2 m}\left(\hat{\mathbf{s}}^{\prime \prime}\right)-B_{l+1}^{-m-1} Y_{l m}\left(\hat{\mathbf{s}}^{\prime \prime}\right)\right] \\
+\frac{3}{8 \pi} g_{l+1} B_{l+1}^{-m-1}\left[B_{l+2}^{m} Y_{l+2 m}\left(\hat{\mathbf{s}}^{\prime \prime}\right)-B_{l+1}^{-m-1} Y_{l m}\left(\hat{\mathbf{s}}^{\prime \prime}\right)\right] \\
\quad+\frac{3}{4 \pi} g_{l-1} A_{l}^{m}\left[A_{l}^{m} Y_{l m}\left(\hat{\mathbf{s}}^{\prime \prime}\right)+A_{l-1}^{m} Y_{l-2 m}\left(\hat{\mathbf{s}}^{\prime \prime}\right)\right] \\
\quad+\frac{3}{4 \pi} g_{l+1} A_{l+1}^{m}\left[A_{l+2}^{m} Y_{l+2 m}\left(\hat{\mathbf{s}}^{\prime \prime}\right)+A_{l+1}^{m} Y_{l m}\left(\hat{\mathbf{s}}^{\prime \prime}\right)\right] \\
\quad-\frac{3}{8 \pi} g_{l-1} B_{l}^{-m}\left[-B_{l}^{-m} Y_{l m}\left(\hat{\mathbf{s}}^{\prime \prime}\right)+B_{l-1}^{m-1} Y_{l-2 m}\left(\hat{\mathbf{s}}^{\prime \prime}\right)\right] \\
\left.+\frac{3}{8 \pi} g_{l+1} B_{l+1}^{m-1}\left[-B_{l+2}^{-m} Y_{l+2 m}\left(\hat{\mathbf{s}}^{\prime \prime}\right)+B_{l+1}^{m-1} Y_{l m}\left(\hat{\mathbf{s}}^{\prime \prime}\right)\right]\right\} .
\end{aligned}
$$

(A10)
The last step is the integral over $\hat{\mathbf{s}}^{\prime \prime}$ which gives the final result for the sensitivity kernel:

$$
\begin{aligned}
K_{1}\left(\mathbf{r}_{b}, \hat{\mathbf{s}}_{b} ; \mathbf{r}_{a}, \hat{\mathbf{s}}_{a} ; \mathbf{r}^{\prime}\right) \\
=\sum_{L=0}^{l_{\max }} \sum_{M=-L}^{L}(-1)^{L+1} g_{L} a_{L M}\left(\mathbf{r}_{2}\right) a_{L M}\left(\mathbf{r}^{\prime}\right) \\
+a_{L M}\left(\mathbf{r}^{\prime}\right)\left\{\frac{1}{2}(-1)^{L} g_{L-1} B_{L}^{M} B_{L}^{M} a_{L M}\left(\mathbf{r}_{2}\right)\right. \\
+\frac{1}{2}(-1)^{L+3} g_{L+1} B_{L+2}^{M} B_{L+1}^{-M-1} a_{L+2 M}\left(\mathbf{r}_{2}\right) \\
+\frac{1}{2}(-1)^{L-1} g_{L-1} B_{L-1}^{-M-1} B_{L}^{M} a_{L-2 M}\left(\mathbf{r}_{2}\right) \\
+\frac{1}{2}(-1)^{L} g_{L+1} B_{L+1}^{-M-1} B_{L+1}^{-M-1} a_{L M}\left(\mathbf{r}_{2}\right) \\
+(-1)^{L} g_{L-1} A_{L}^{M} A_{L}^{M} a_{L M}\left(\mathbf{r}_{2}\right) \\
+(-1)^{L+2} g_{L+1} A_{L+2}^{M} A_{L+1}^{M} a_{L+2 M}\left(\mathbf{r}_{2}\right) \\
+(-1)^{L-2} g_{L-1} A_{L-1}^{M} A_{L}^{M} a_{L-2 M}\left(\mathbf{r}_{2}\right) \\
+(-1)^{L} g_{L+1} A_{L+1}^{M} A_{L+1}^{M} a_{L M}\left(\mathbf{r}_{2}\right) \\
+\frac{1}{2}(-1)^{L} g_{L-1} B_{L}^{-M} B_{L}^{-M} a_{L M}\left(\mathbf{r}_{2}\right) \\
+\frac{1}{2}(-1)^{L+3} g_{L+1} B_{L+2}^{-M} B_{L+1}^{M-1} a_{L+2 M}\left(\mathbf{r}_{2}\right) \\
+\frac{1}{2}(-1)^{L-1} g_{L-1} B_{L-1}^{M-1} B_{L}^{-M} a_{L-2 M}\left(\mathbf{r}_{2}\right) \\
\left.+(-1)^{L+1} g_{L+1} B_{L+1}^{M-1} B_{L+1}^{M-1} a_{L M}\left(\mathbf{r}_{2}\right)\right\} \\
\\
+
\end{aligned}
$$

where to simplify the notation we set $L=l^{\prime \prime \prime}$ and $M=m^{\prime \prime \prime}$. The Monte Carlo model as in Ref. [26] is used to calculate the Green's functions and the expansion in spherical harmonics with coefficients $a_{L M}$ up to $l_{\max }=15$. The translational invariance is then used so that one coefficient is evaluated at the position $\mathbf{r}_{2}$ which is just the position of the source translated by the vector joining the source and detector. Hence, the expression of (A11) can be evaluated in postprocessing, i.e., just reading the output coefficients of the Monte Carlo model and performing the sum over $L$ and $M$. It is also worth noting that the same kernel $K_{1}$ can be used for DCT and SCOT.
[1] S. R. Arridge, Inverse Probl. 15, R41 (1999).

[2] D. A. Boas, D. H. Brooks, E. L. Miller, C. A. DiMarzio, M. Kilmer, R. J. Gaudette, and Q. Zhang, IEEE Signal Proc. Mag. 18, 57 (2001).

[3] S. R. Arridge and J. C. Schotland, Inverse Probl. 25, 123010 (2009).

[4] D. A. Boas, L. E. Campbell, and A. G. Yodh, Phys. Rev. Lett. 75, 1855 (1995).

[5] D. Boas and A. Yodh, J. Opt. Soc. Am. A 14, 192 (1997).

[6] T. Durduran, R. Choe, W. B. Baker, and A. G. Yodh, Rep. Prog. Phys. 73, 076701 (2010).
[7] R. Pecora, Dynamic Light Scattering: Applications of Photon Correlation Spectroscopy (Springer Science \& Business Media, 2013).

[8] G. Maret and P. Wolf, Z. Phys. B 65, 409 (1987).

[9] F. C. MacKintosh and S. John, Phys. Rev. B 40, 2383 (1989).

[10] R. Carminati, R. Elaloufi, and J.-J. Greffet, Phys. Rev. Lett. 92, 213903 (2004).

[11] T. Durduran and A. G. Yodh, NeuroImage 85, 51 (2014).

[12] C. Zhou, G. Yu, D. Furuya, J. H. Greenberg, A. G. Yodh, and T. Durduran, Opt. Express 14, 1125 (2006).

[13] D. A. Boas and A. K. Dunn, J. Biomed. Opt. 15, 011109 (2010). 
[14] A. Mazhar, D. J. Cuccia, T. B. Rice, S. A. Carp, A. J. Durkin, D. A. Boas, B. Choi, and B. J. Tromberg, Biomed. Opt. Express 2, 1553 (2011).

[15] T. B. Rice, S. D. Konecky, A. Mazhar, D. J. Cuccia, A. J. Durkin, B. Choi, and B. J. Tromberg, J. Opt. Soc. Am. A 28, 2108 (2011).

[16] H. M. Varma, C. P. Valdes, A. K. Kristoffersen, J. P. Culver, and T. Durduran, Biomed. Opt. Express 5, 1275 (2014).

[17] T. Dragojević, H. M. Varma, J. L. Hollmann, C. P. Valdes, J. P. Culver, C. Justicia, and T. Durduran, NeuroImage 153, 283 (2017).

[18] P. Kaplan, M. H. Kao, A. Yodh, and D. J. Pine, Appl. Opt. 32, 3828 (1993).

[19] R. Pierrat, N. B. Braham, L. F. Rojas-Ochoa, R. Carminati, and F. Scheffold, Opt. Commun. 281, 18 (2008).

[20] L. F. Rojas-Ochoa, D. Lacoste, R. Lenke, P. Schurtenberger, and F. Scheffold, J. Opt. Soc. Am. A 21, 1799 (2004).
[21] P. Zakharov, A. Völker, A. Buck, B. Weber, and F. Scheffold, Opt. Lett. 31, 3465 (2006)

[22] B. Ackerson, R. Dougherty, N. Reguigui, and U. Nobbmann, J. Thermophys. Heat Transfer 6, 577 (1992).

[23] R. Dougherty, B. Ackerson, N. Reguigui, F. Dorri-Nowkoorani, and U. Nobbmann, J. Quant. Spectrosc. Radiat. Transfer 52, 713 (1994).

[24] J. C. Schotland and V. A. Markel, Inverse Probl. Imaging 1, 181 (2007).

[25] D. A. Boas, Opt. Express 1, 404 (1997).

[26] U. Tricoli, C. M. Macdonald, A. Da Silva, and V. A. Markel, Opt. Lett. 42, 362 (2017).

[27] C. M. Macdonald, U. Tricoli, A. Da Silva, and V. A. Markel, J. Opt. Soc. Am. A 34, 1330 (2017).

[28] U. Tricoli, C. M. Macdonald, A. Da Silva, and V. A. Markel, J. Opt. Soc. Am. A 35, 356 (2018). 